Original Research Paper

\title{
Common Fixed Point Theorems for Four Maps in G-Partial Metric Spaces
}

\author{
Kanayo Stella Eke and Grace O. Akinlabi \\ Department of Mathematics, Covenant University, Canaanland, Ota, Nigeria
}

\author{
Article history \\ Received: 18-08-2016 \\ Revised: 01-02-2017 \\ Accepted: 09-03-2017 \\ Corresponding Author: \\ Kanayo Stella Eke \\ Department of Mathematics, \\ Covenant University, \\ Canaanland, Ota, Nigeria \\ Email: \\ kanayo.eke@covenantuniversity.edu.ng
}

\begin{abstract}
The common fixed point principle for two set of maps satisfying specified contractive conditions in cone metric spaces is proved in the context of G-partial metric space and none of the maps involved therein is continuous. Our research outcome extends well known similar results available in the literature.
\end{abstract}

Keywords: Common Fixed Points, Generalized Contraction Maps, G-Partial Metric Space

\section{Introduction}

The establishment of common fixed point principle was initiated by Jungck (1976). In the same reference, the commutativity of one pair of maps was introduced. Sessa (1982) later introduced weak commutativity of maps. Jungck (1986) improved on this by initiating compatibility. Furthermore, weak compatibility was inaugurated by Jungck (1996). Many researchers employed the concept of weakly compatible maps currently for proving common fixed point theorems in various spaces. Existence and uniqueness of common fixed points of constrictive maps was first proved in metric spaces. Subsequently, many authors developed interest in this area and enlarged the space. Matthews (1992) extended the metric space to partial metric space by including the non-zero self-distance to the assumption of metric spaces. Additionally, the literature on metric space has also been improved by Mustafa and Sims (2006) where every triplet of an arbitrary set is being given as real numbers. The general view of G-metric space has additionally been improved by introducing partial metric space to the condition of G-metric space leading to G-partial metric space (Eke and Olaleru, 2013). The authors proved that Banach constriction principle has unique fixed point in ordered G-partial metric spaces.

The literature has also been enriched with new theorems on fixed and common fixed point of contractive and expansive operators in this space. For instance, fixed point of ciric-type constrictive operators was proved in ordered G-partial metric spaces (Olaleru et al., 2014). Eke (2015) establishes some fixed point theorems for two expansive operators in G-partial metric spaces. Existence of coincidence point for contractive operators in generalized metric space is proved (Abbas and Rhoades, 2009). The existence of common fixed point for four mappings that satisfy generalized contractive conditions in cone metric space without allowing the continuity of any of the maps has also been proved (Abbas et al., 2010).

Motivated by these results, we establish some common fixed point theorems for two set of operators satisfying certain enlarged contractive conditions in Gpartial metric spaces.

Consistent with Eke and Olaleru (2013), we present the following definitions and results.

\section{Definition 1.1}

Suppose $A$ is a nonempty set and further if $G_{p}: A \times A \times A \rightarrow \mathbb{R}^{+}$is a function fulfilling the following:

- $\left(G_{p} 1\right) G_{p}(a, b, c) \geq G_{p}(a, a, a) \geq 0$ for all $a, b, c \in A$ (small self-distance)

- $\left(G_{p} 2\right) G_{p}(a, b, c)=G_{p}(a, a, b)=G_{p}(b, b, c)=G_{p}(c, c, a)$

if and only if $a=b=c$, (equality)

- $\quad\left(G_{p} 3\right) G_{p}(a, b, c)=G_{p}(c, a, b)=G_{p}(b, c, a) \quad$ (symmetry in all three variables)

- $\left(G_{p} 4\right) G_{p}(a, b, c) \leq G_{p}(a, e, e)+G_{p}(e, b, c)-G_{p}(e, e, e)$ (re ctangle inequality)

Then we have G-partial metric space which comprises of the set $\left(A, G_{p}\right)$, where $G_{p}$ is called Gpartial metric. 


\section{Example 1.2}

Suppose $A=\mathbb{R}^{+}$and $G_{p}: A \times A \times A \rightarrow \mathbb{R}^{+}$is a G-partial metric characterized by $G_{p}(a, b, c)=\max \{a, b, c\}$ then $\left(A, G_{p}\right)$ is a G-partial metric space.

Introducing the following:

\section{Definition 1.3}

If a sequence $\left\{a_{n}\right\}$ of points in a G- partial metric space $\left(A, G_{p}\right)$ has a limit at a point $e \in A$ then we have $\lim _{n \rightarrow \infty} G_{p}\left(a_{n}, a_{n}, e\right)=\lim _{n \rightarrow \infty} G_{p}\left(a_{n}, a_{n}, a_{n}\right)=G_{p}(e, e, e)$.

\section{Definition 1.4}

A sequence $\left\{a_{n}\right\}$ of points in a G-partial metric space $\left(A, G_{p}\right)$ is Cauchy if the number $G_{p}\left(a_{n}, a_{m}, a_{l}\right)$ converges to some $e \in A$ as $n, m, l$ approach infinity.

The proof of the following proposition easily follows from definition.

\section{Proposition 1.5}

Let $\left\{a_{n}\right\}$ be a sequence in G-partial metric space $A$ and $e \in A$. If $\left\{a_{n}\right\}$ converges to $e \in A$, then $\left\{a_{n}\right\}$ is a Cauchy sequence.

\section{Definition 1.6}

A G-partial metric space $\left(A, G_{p}\right)$ is said to be complete if every Cauchy sequence in $\left(A, G_{p}\right)$ converges to an element in $\left(A, G_{p}\right)$. That is, $G_{p}(a, a, a)=\lim _{n \rightarrow \infty} G_{p}\left(a_{n}, a, a\right)=\lim _{n, m \rightarrow \infty} G_{p}\left(a_{n}, a_{m}, a_{m}\right)$.

\section{Definition 1.7}

Let $h$ and $j$ be self-maps on a set $A$. If $u=h a=j a$, for some $a$ in $A$, then $a$ is called coincidence point of $h$ and $j$. Where $u$ is called a point of coincidence of $h$ and $j$.

\section{Definition 1.8}

Let $h$ and $j$ be two self-maps defined on a set $A$. Then $h$ and $j$ are said to be weakly compatible if they commute at every coincidence point.

\section{Main Results}

\section{Theorem 2.1}

Let $h, j, K$ and $L$ be self-maps of a G-partial metric space $A$ satisfying $h(A) \subset L(A), j(A) \subset K(A)$ and $G_{p}(h a, h a$, $j b) \leq q u_{a, a, b}(h, j, K, L)$, where $q \in(0,1)$.

and:

$$
u_{a, a, b}(h, j, K, L) \in\left\{\begin{array}{l}
G_{p}(K a, K a, L b), G_{p}(h a, h a, K a), \\
G_{p}(j b, j b, L b), \\
\frac{G_{p}(h a, h a, L b)+G_{p}(j b, j b, K a)}{2}
\end{array}\right\}
$$

and:

$$
u_{a, b, b}(h, j, K, L) \in\left\{\begin{array}{l}
G_{p}(K a, L b, L b), G_{p}(h a, K a, K a), \\
G_{p}(j b, K b, K b), \\
\frac{G_{p}(h a, L b, L b)+G_{p}(j b, K a, K a s)}{2}
\end{array}\right\}
$$

for all and $a, b \in A$. If one of $h(A), j(A), K(A)$ or $L(A)$ is a complete subspace of $A$, then $\{h, K\}$ and $\{j, L\}$ have a unique point of coincidence in $A$. Moreover, if $\{h, K\}$ and $\{j, L\}$ are weakly compatible, then $h, j, K$ and $L$ have a unique common fixed point.

\section{Proof}

For any arbitrary point $a_{0} \in A$, since $h(A) \subset L(A)$ then there exists $a_{1} \in A$ such that $h a_{0} \subset L a_{1}$. If the range of $j(A)$ is contained in the range of $K(A)$ then there exists $a_{2} \in A$ such that $j a_{1} \subset K a_{2}$. Continue the process, we can construct two sequences $\left\{a_{n}\right\}$ and $\left\{a_{n}\right\}$ in $A$ such that:

$$
\begin{aligned}
& h a_{2 k}=L a_{2 k+1}=b_{2 k+1} \\
& j a_{2 k+1}=K a_{2 k+2}=b_{2 k+2}
\end{aligned}
$$

For a given $n \in N$, if $n$ is even, say $n=2 k$ for some $k \in N$ then, using (1), we obtain:

$$
\begin{aligned}
G_{p}\left(b_{n+1}, b_{n+1}, b_{n}\right) & =G_{p}\left(b_{2 k+1}, b_{2 k+1}, b_{2 k}\right) \\
& =G_{p}\left(h a_{2 k}, h a_{2 k}, j a_{2 k-1}\right) \\
& \leq u_{a, a, b}(h, j, K, L), n \geq 1
\end{aligned}
$$

where:

$$
\begin{aligned}
& u_{a, a, b}(h, j, K, L) \\
& \in\left\{\begin{array}{l}
G_{p}\left(K a_{2 k}, K a_{2 k}, L a_{2 k-1}\right), G_{p}\left(h a_{2 k}, h a_{2 k}, K a_{2 k}\right), \\
G_{p}\left(j a_{2 k-1}, j a_{2 k-1}, L a_{2 k-1}\right), \\
\frac{G_{p}\left(h a_{2 k}, h a_{2 k}, L a_{2 k-1}\right)+G_{p}\left(j a_{2 k-1}, j a_{2 k-1}, K a_{2 k}\right)}{2}
\end{array}\right\} \\
& =\left\{\begin{array}{l}
G_{p}\left(b_{2 k}, b_{2 k}, b_{2 k-1}\right), G_{p}\left(b_{2 k+1}, b_{2 k+1}, b_{2 k}\right), \\
\left.\frac{G_{p}\left(b_{2 k+1}, b_{2 k}, b_{2 k-1}\right),}{2}, b_{2 k-1}\right)+G_{p}\left(b_{2 k}, b_{2 k}, b_{2 k}\right) \\
2
\end{array}\right\} \\
& \leq\left\{\begin{array}{l}
\frac{G_{p}\left(b_{2 k}, b_{2 k}, b_{2 k-1}\right), G_{p}\left(b_{2 k+1}, b_{2 k+1}, b_{2 k}\right), G_{p}\left(b_{2 k}, b_{2 k}, b_{2 k-1}\right)}{\frac{G_{p}\left(b_{2 k+1}, b_{2 k+1}, b_{2 k}\right)+G_{p}\left(b_{2 k}, b_{2 k}, b_{2 k-1}\right)}{2}} \\
\frac{-G_{p}\left(b_{2 k}, b_{2 k}, b_{2 k}\right)+G_{p}\left(b_{2 k}, b_{2 k}, b_{2 k}\right)}{2}
\end{array}\right\}
\end{aligned}
$$




$$
\begin{aligned}
& =\left\{\begin{array}{l}
G_{p}\left(b_{2 k}, b_{2 k}, b_{2 k-1}\right), G_{p}\left(b_{2 k+1}, b_{2 k+1}, b_{2 k}\right), \\
G_{p}\left(b_{2 k}, b_{2 k}, b_{2 k-1}\right), \\
\frac{G_{p}\left(b_{2 k+1}, b_{2 k+1}, b_{2 k}\right)+G_{p}\left(b_{2 k}, b_{2 k}, b_{2 k-1}\right)}{2}
\end{array}\right\} \\
& =\left\{\begin{array}{l}
G_{p}\left(b_{n}, b_{n}, b_{n-1}\right), G_{p}\left(b_{n+1}, b_{n+1}, b_{n}\right), \\
G_{p}\left(b_{n}, b_{n}, b_{n-1}\right), \\
\frac{G_{p}\left(b_{n+1}, b_{n+1}, b_{n}\right)+G_{p}\left(b_{n}, b_{n}, b_{n-1}\right)}{2}
\end{array}\right\}
\end{aligned}
$$

If $u_{a, a, b}(h, j, K, L)=G_{p}=\left(b_{n}, b_{n}, b_{n-1}\right)$ then $G_{p}=\left(b_{n+1}\right.$, $\left.b_{n+1}, b_{n}\right) \leq q G_{p}\left(b_{n}, b_{n}, b_{n-1}\right)$ If $u_{x, x, y}(h, j, K, L)=G_{p}\left(b_{n+1}\right.$, $\left.b_{n+1}, b_{n}\right)$ then $G_{p}=\left(b_{n+1}, b_{n+1}, b_{n}\right) \leq q G_{p}\left(b_{n}, b_{n}, b_{n-1}\right)$, a contradiction.

$$
\begin{aligned}
& u_{a, a, b}(h, j, K, L)=\frac{G_{p}\left(b_{n+1}, b_{n+1}, b_{n}\right)+G_{p}\left(b_{n}, b_{n}, b_{n-1}\right)}{2} \text { then: } \\
& G_{p}\left(b_{n+1}, b_{n+1}, b_{n}\right) \leq \frac{q}{2}\left[G_{p}\left(b_{n+1}, b_{n+1}, b_{n}\right)+G_{p}\left(b_{n}, b_{n}, b_{n-1}\right)\right] \\
& \left(1-\frac{q}{2}\right) G_{p}\left(b_{n+1}, b_{n+1}, b_{n}\right) \leq \frac{q}{2}\left[G_{p}\left(b_{n}, b_{n}, b_{n-1}\right)\right] \\
& \left(\frac{2-q}{2}\right) G_{p}\left(b_{n+1}, b_{n+1}, b_{n}\right) \leq \frac{q}{2}\left[G_{p}\left(b_{n}, b_{n}, b_{n-1}\right)\right] \\
& (2-q) G_{p}\left(b_{n+1}, b_{n+1}, b_{n}\right) \leq 2\left[G_{p}\left(b_{n}, b_{n}, b_{n-1}\right)\right] \\
& G_{p}\left(b_{n+1}, b_{n+1}, b_{n}\right) \leq \frac{q}{2-q}\left[G_{p}\left(b_{n}, b_{n}, b_{n-1}\right)\right] \\
& \text { Let } \delta=\max \left\{q, \frac{q}{2-q}\right\}<1, \text { then: }
\end{aligned}
$$

$$
G_{p}\left(b_{n+1}, b_{n+1}, b_{n}\right) \leq \delta G_{p}\left(b_{n}, b_{n}, b_{n-1}\right)
$$

If $n$ is odd, so $n=2 k+1$ for some $k \in N$. Then using (1) we obtain:

$$
\begin{aligned}
G_{p}\left(b_{n+1}, b_{n+1}, b_{n}\right) & =G_{p}\left(b_{2 k+2}, b_{2 k+2}, b_{2 k+1}\right) \\
& =G_{p}\left(h a_{2 k+1}, h a_{2 k+1}, j a_{2 k}\right) \\
& \leq u_{a, a, b}(h, j, K, L), n \geq 1
\end{aligned}
$$

where:

$$
\begin{aligned}
& u_{a, a, b}(h, j, K, L) \\
& \in\left\{\begin{array}{l}
G_{p}\left(K a_{2 k+1}, K a_{2 k+1}, L a_{2 k}\right), G_{p}\left(h a_{2 k+1}, h a_{2 k+1}, K a_{2 k+1}\right) \\
G_{p}\left(j a_{2 k}, j a_{2 k}, L a_{2 k}\right), \\
\frac{G_{p}\left(h a_{2 k+1}, h a_{2 k+1}, L a_{2 k}\right)+G_{p}\left(j a_{2 k}, j a_{2 k}, K a_{2 k+1}\right)}{2}
\end{array}\right\}
\end{aligned}
$$

$$
\begin{aligned}
& =\left\{\begin{array}{l}
G_{p}\left(b_{2 k+1}, b_{2 k+1}, b_{2 k}\right), G_{p}\left(b_{2 k+2}, b_{2 k+2}, b_{2 k+1}\right), \\
G_{p}\left(b_{2 k+1}, b_{2 k+1}, b_{2 k}\right), \\
\frac{G_{p}\left(b_{2 k+2}, b_{2 k+2}, b_{2 k}\right)+G_{p}\left(b_{2 k+1}, b_{2 k+1}, b_{2 k+1}\right)}{2}
\end{array}\right\} \\
& \leq\left\{\begin{array}{l}
\frac{G_{p}\left(b_{2 k+1}, b_{2 k+1}, b_{2 k}\right), G_{p}\left(b_{2 k+2}, b_{2 k+2}, b_{2 k+1}\right), G_{p}\left(b_{2 k+1}, b_{2 k+1}, b_{2 k}\right),}{2}\left(b_{2 k+2}, b_{2 k+2}, b_{2 k+1}\right)+G_{p}\left(b_{2 k+1}, b_{2 k+1}, b_{2 k}\right) \\
\frac{-G_{p}\left(b_{2 k+1}, b_{2 k+1}, b_{2 k+1}\right)+G_{p}\left(b_{2 k+1}, b_{2 k+1}, b_{2 k+1}\right)}{2}
\end{array}\right\} \\
& =\left\{\begin{array}{l}
G_{p}\left(b_{2 k+1}, b_{2 k+1}, b_{2 k}\right), G_{p}\left(b_{2 k+2}, b_{2 k+2}, b_{2 k+1}\right), \\
G_{p}\left(b_{2 k+1}, b_{2 k+1}, b_{2 k}\right), \\
\frac{G_{p}\left(b_{2 k+2}, b_{2 k+2}, b_{2 k+1}\right)+G_{p}\left(b_{2 k+1}, b_{2 k+1}, b_{2 k}\right)}{2}
\end{array}\right\} \\
& =\left\{\begin{array}{l}
G_{p}\left(b_{n}, b_{n}, b_{n-1}\right), G_{p}\left(b_{n+1}, b_{n+1}, b_{n}\right), \\
G_{p}\left(b_{n}, b_{n}, b_{n-1}\right), \\
\frac{G_{p}\left(b_{n+1}, b_{n+1}, b_{n}\right)+G_{p}\left(b_{n}, b_{n}, b_{n-1}\right)}{2}
\end{array}\right\}
\end{aligned}
$$

Following the similar argument as above we obtain:

$G_{p}\left(b_{n+1}, b_{n+1}, b_{n}\right) \leq \delta G_{p}\left(b_{n}, b_{n}, b_{n-1}\right)$

Consequently, we obtain:

$$
G_{p}\left(b_{n+1}, b_{n+1}, b_{n}\right) \leq \delta^{n} G_{p}\left(b_{1}, b_{1}, b_{0}\right)
$$

For $m>n$ and using the rectangle inequality, we get:

$$
\begin{aligned}
G_{p}\left(b_{n}, b_{n}, b_{m}\right) \leq & G_{p}\left(b_{n}, b_{n}, b_{n+1}\right)+G_{p}\left(b_{n+1}, b_{n+1}, b_{n+2}\right) \\
& +G_{p}\left(b_{n+2}, b_{n+2}, b_{n+3}\right)+\cdots+G_{p}\left(b_{m-1}, b_{m}, b_{m}\right) \\
& -G_{p}\left(b_{n+1}, b_{n+1}, b_{n+2}\right)-\cdots-G_{p}\left(b_{m-1}, b_{m-1}, b_{m-1}\right) \\
& \leq G_{p}\left(b_{n}, b_{n}, b_{n+1}\right)+G_{p}\left(b_{n+1}, b_{n+1}, b_{n+2}\right) \\
& +G_{p}\left(b_{n+2}, b_{n+2}, b_{n+3}\right)+\cdots+G_{p}\left(b_{m-1}, b_{m}, b_{m}\right) \\
& \leq \delta^{n} G_{p}\left(b_{0}, b_{0}, b_{1}\right)+\delta^{n+1} G_{p}\left(b_{0}, b_{0}, b_{1}\right) \\
& +\cdots+\delta^{m-1} G_{p}\left(b_{0}, b_{0}, b_{1}\right) \\
& \leq\left(\delta^{n}+\delta^{n+1}+\cdots+\delta^{m-1}\right) G_{p}\left(b_{0}, b_{0}, b_{1}\right) \\
& \leq \delta^{n}\left(1+\delta+\cdots+\delta^{m-n-1}\right) G_{p}\left(b_{0}, b_{0}, b_{1}\right) \\
& \leq \frac{\delta^{n}}{1-\delta} G_{p}\left(b_{0}, b_{0}, b_{1}\right) .
\end{aligned}
$$

This shows that $G_{p}\left(b_{n}, b_{n}, b_{m}\right)$ converges to zero as $\mathrm{m}$ and $\mathrm{n}$ tend to infinity. Thus $\left\{b_{n}\right\}$ is a Cauchy sequence in $A$. Suppose $K(A)$ is complete, then there exists a $u$ in $K(A)$ such that $K a_{2 k}=b_{2 k} \rightarrow u$ as $k \rightarrow \infty$. It is equivalent to 
$K a_{2 k}=j a_{2 k-1}=b_{2 k} \rightarrow u$ and $b 2_{k-1}=L a_{2 k-1}=h a_{2 k-2} \rightarrow u$ as $k \rightarrow \infty$.

Also

$G_{p}(u, u, u)=\lim _{k \rightarrow \infty} G_{p}\left(b_{2 k}, u, u\right)=\lim _{m, n \rightarrow \infty} G_{p}\left(b_{n}, b_{n}, b_{n}\right)=0$.

is,

Suppose there is $v$ in $A$ such that $K v=u$. Then we show that $h v=u$. On the contrary, we have:

$$
\begin{aligned}
G_{p}(h v, h v, u) & \leq G_{p}\left(h v, h v, j a_{2 k-1}\right)+G_{p}\left(j a_{2 k-1}, j a_{2 k-1}, u\right) \\
& -G_{p}\left(j a_{2 k-1}, j a_{2 k-1}, j a_{2 k-1}\right) \\
& \leq G_{p}\left(h v, h v, j a_{2 k-1}\right)+G_{p}\left(j a_{2 k-1}, j a_{2 k-1}, u\right) \\
& \leq q u_{v, v, j a}(h, j, K, L)+G_{p}\left(j a_{2 k-1}, j a_{2 k-1}, u\right)
\end{aligned}
$$

where:

$$
u_{v, v, a_{2 k-1}}(h, j, K, L) \in\left\{\begin{array}{l}
G_{p}\left(K v, K v, L a_{2 k-1}\right), G_{p}(h v, h v, K v), \\
G_{p}\left(j a_{2 k-1}, j a_{2 k-1}, L a_{2 k-1}\right), \\
\frac{G_{p}\left(h v, h v, L a_{2 k-1}\right)+G_{p}\left(h a_{2 k-1}, h a_{2 k-1}, K v\right)}{2}
\end{array}\right\}
$$

for $k \in N$. Minimum of one from four possible elements could result from the set $u_{v, v, a 2 k-1}(h, j, K, L)$ many times. Hence, four possible outcome can occur.

If $u_{v, v, a 2 k-1}(h, j, K, L)=G_{p}\left(K v, K v, L a_{2 k-1}\right)$ then we have $G_{p}(h v, h v, u) \leq q G_{p}\left(K v, K v, L a_{2 k-1}\right)+G_{p}\left(j a_{2 k-1}, j a_{2 k-1}\right.$, $u)$. As $k \rightarrow \infty$ we obtain $G_{p}(h v, h v, u) \leq 0$. This implies that $G_{p}(h v, h v, u)=0$.

If $u_{v, v, a 2 k-1}(h, j, K, L)=G_{p}(h v, h v, K v)$ then we have $G_{p}(h v, h v, u) \leq q G_{p}(h v, h v, K v)+\left(j a_{2 k-1}, j a_{2 k-1}, u\right)$. As $k \rightarrow \infty$ we get $G_{p}(h v, h v, u) \leq q G_{p}(h v, h v, K v)=q G_{p}(h v$, $h v, u)$, a contradiction.

If $u_{v, v, a 2 k-1}(h, j, K, L)=G p\left(j a_{2 k-1}, j a_{2 k-1}, L a_{2 k-1}\right)$ then we have $G_{p}(h v, h v, u) \leq q G p\left(j a_{2 k-1}, j a_{2 k-1}, L a_{2 k-1}\right)+G_{p}\left(j a_{2 k-}\right.$ $\left.{ }_{1}, j a_{2 k-1}, u\right)$. As $k \rightarrow \infty$ we get $G_{p}(h v, h v, u) \leq 0$. This implies that $G_{p}(h v, h v, \quad u)=0$. If $u_{v, v, j a_{2 k-1}}(h, j, K, L)=\frac{G_{p}(h v, h v, u)+G_{p}\left(j a_{2 k-1}, j a_{2 k-1}, K v\right)}{2}$

then we have $G_{p}(h v, h v, u) \leq q / 2\left\{G_{p}\left(h v, h v, L a_{2 k-}\right.\right.$ $\left.\left.{ }_{1}\right)+G_{p}\left(j a_{2 k-1}, j a_{2 k-1}, K v\right)+G_{p}\left(j a_{2 k-1}, j a_{2 k-1}, u\right)\right\}$. As $k \rightarrow \infty$ we get $G_{p}(h v, h v, u) \leq q / 2 G_{p}(h v, h v, u)$, a contradiction.

Thus, in all four cases we have that $\mathrm{G}_{\mathrm{p}}(h v, h v, u)=0$. This implies that $u=h_{v}=k_{v}$. Therefore $u$ is a point of coincidence of $h$ and $K$. Since $u \in h(A) \subset L(A)$, then there exists a $w \in A$ such that $L w=u$. We claim that $j w=u$. On the contrary, let $j w \neq u$, then using (1) we have:

$$
\begin{aligned}
G_{p}(u, u, j w) & \leq G_{p}\left(u, u, h a_{2 k}\right)+G_{p}\left(h a_{2 k}, h a_{2 k}, j w\right) \\
& -G_{p}\left(h a_{2 k}, h a_{2 k}, h a_{2 k}\right) \\
& \leq G_{p}\left(u, u, h a_{2 k}\right)+G_{p}\left(h a_{2 k}, h a_{2 k}, j w\right) \\
& \leq G_{p}\left(u, u, h a_{2 k}\right)+q u_{2 k, 2 k, w}(h, j, K, L)
\end{aligned}
$$

where:

$$
u_{2 k, 2 k, w}(h, j, K, L) \in\left\{\begin{array}{l}
G_{p}\left(K a_{2 k}, K a_{2 k}, L w\right), G_{p}\left(h a_{2 k}, h a_{2 k}, K a_{2 k}\right), \\
G_{p}(j w, j w, L w), \\
\frac{G_{p}\left(h a_{2 k}, h a_{2 k}, L w\right)+G_{p}\left(j w, j w, K a_{2 k}\right)}{2}
\end{array}\right\}
$$

for $k \in N$. Minimum of one from four possible elements could result from the set $u_{v, v, a 2 k-1}(h, j, K, L)$ many times. Hence, four possible outcome can occur.

If $u_{2 k, 2 k, w}(h, j, K, L)=\left(K a_{2 k}, K a_{2 k}, L w\right)$ then we have $G_{p}(u, u, j w) \leq G p\left(u, u, h a_{2 k}\right)+q G_{p}\left(K a_{2 k}, K a_{2 k}, L w\right)$. As $k \rightarrow \infty$ we obtain $G_{p}(u, u, j w) \leq 0$. This implies that $G_{p}(u, u, j w)=0$.

If $u_{2 k, 2 k, w}(h, j, K, L)=G_{p}\left(h a_{2 k}, h a_{2 k}, K a_{2 k}\right)$ then we have $G_{p}(u, u, j w) \leq G p\left(u, u, h a_{2 k}\right)+q G_{p}(j w, j w, L w)$. As $k \rightarrow \infty$ we obtain $G_{p}(u, u, j w) \leq q G p(j w, j w, u)=q G_{p}(u, j w, j w)$.

If

$$
u_{2 k, 2 k, w}(h, j, K, L)=
$$

$\frac{G_{p}\left(h a_{2 k}, h a_{2 k}, L w\right)+G_{p}\left(j w, j w, K a_{2 k}\right)}{2}$ then we have $G_{p}(u$, $u, j w) \leq G_{p}\left(u, u, h a_{2 k}\right)+q / 2\left\{G_{p}\left(h a_{2 k}, h a_{2 k}, L w\right)+G_{p}(j w, j w\right.$, $L w)\}$. As $k \rightarrow \infty$ we obtain $G_{p}(u, u, j w) \leq \frac{q}{2} G_{p}(j w, j w, u)=\frac{q}{2} G_{p}(u, j w, j w)$.

Let $\max \left\{q, \frac{q}{2}\right\}=q$. Then:

$G_{p}(u, u, j w) \leq q G_{p}(u, j w, j w)$

Also using (2) we obtain the following:

$$
\begin{aligned}
G_{p}(u, u, j w) & \leq G_{p}\left(u, h a_{2 k}, h a_{2 k}\right) \\
& +G_{p}\left(h a_{2 k}, j w, j w\right)-G_{p}\left(h a_{2 k}, h a_{2 k}, h a_{2 k}\right) \\
& \leq G_{p}\left(u, h a_{2 k}, h a_{2 k}\right)+G_{p}\left(h a_{2 k}, j w, j w\right) \\
& \leq G_{p}\left(u, h a_{2 k}, h a_{2 k}\right)+q u_{2 k, w, w}(h, j, K, L)
\end{aligned}
$$

where:

$$
\begin{aligned}
& u_{a_{2 k}, w, w}(h, j, K, L) \in\left\{\begin{array}{l}
G_{p}\left(K a_{2 k}, L w, L w\right), G_{p}\left(h a_{2 k}, K a_{2 k}, K a_{2 k}\right), \\
G_{p}(j w, L w, L w), \\
\frac{G_{p}\left(h a_{2 k}, L w, L w\right)+G_{p}\left(j w, K a_{2 k}, K a_{2 k}\right)}{2}
\end{array}\right\} \\
& \text { If } \quad u_{a_{2 k}, w, w}(h, j, K, L)=G_{p}\left(K a_{2 k}, L w, L w\right) \quad \text { then } \\
& \begin{array}{ll}
G_{p}(u, j w, j w) \leq G_{p}\left(u, h a_{2 k}, h a_{2 k}\right)+q G_{p}\left(K a_{2 k}, L w, L w\right) . & \text { As } \\
k \rightarrow \infty \quad \text { we have } G_{p}(u, j w, j w) \leq 0 \text {. This implies that } &
\end{array} \\
& G_{p}(u, j w, j w)=0 .
\end{aligned}
$$


If $u_{a 2 k, w, w}(h, j, K, L)=G_{p}\left(h a_{2 k}, K a_{2 k}, K a_{2 k}\right)$ then $G_{p}(u, j w, j w) \leq G_{p}\left(u, h a_{2 k}, h a_{2 k}\right)+q G_{p}\left(h a_{2 k}, h a_{2 k}, K a_{2 k}\right)$. As $k \rightarrow \infty$ we have $G_{p}(u, j w, j w) \leq 0$. This implies that $G_{p}(u, j w, j w)=0$.

If $u_{a 2 k, w, w}(h, j, K, L)=G_{p}(j w, L w, L w)$ then $G_{p}(u, j w$, $j w) \leq G_{p}\left(u, h a_{2 k}, h a_{2 k}\right)+q G_{p}(j w, L w, L w)$. As $k \rightarrow \infty$ we have $G_{p}(u, j w, j w) \leq \mathrm{q} G_{p}(j w, u, u)=q G_{p}(u, u, j w)$.

If

$\frac{G_{p}\left(h a_{2 k}, L w, L w\right)+G_{p}\left(j w, K a_{2 k}, K a_{2 k}\right)}{2}$ then $\mathrm{G}_{\mathrm{p}}(u, j w$, $j w) \leq G_{p}\left(u, h a_{2 k}, h a_{2 k}\right)+q / 2\left\{G_{p}\left(h a_{2 k}, L w, L w\right)+G_{p}\left(j w, K a_{2 k}\right.\right.$, $\left.\left.K a_{2 k}\right)\right\}$. As $k \rightarrow \infty$ we have $G_{p}(u, j w, j w) \leq \frac{q}{2} G_{p}(j w, u, u)=\frac{q}{2} G_{p}(u, u, j w)$.

Let $\max \left\{q, \frac{q}{2}\right\}=q$. Then:

$G_{p}(u, j w, j w) \leq q G_{p}(u, u, j w)$

Combining (3) and (4) yields:

$$
G_{p}(u, u, j w) \leq q G_{p}(u, j w, j w) \leq q^{2} G_{p}(u, u, j w)
$$

This is a contradiction since $q<1$, hence $u=j w=L w$. Thus $\{h, K\}$ and $\{j, L\}$ have common point of coincidence in $A$. Now, if $\{h, K\}$ and $\{j, L\}$ are weakly compatible then $h u=h K v=K h v=K u=w_{1}$ (say) and $j u$ $=j L v=L j v=L u w_{2}$ (say).

Now we show that the points of coincidence are unique:

$$
G_{p}\left(w_{1}, w_{2}, w_{3}\right) \leq G_{p}(h u, h u, j u) \leq q u_{u, u, u}(h, j, K, L)
$$

where:

$$
\begin{aligned}
& u_{u, u, u}(h, j, K, L) \\
& \in\left\{\begin{array}{l}
G_{p}(K u, K u, L u), G_{p}(h u, h u, K u), G_{p}(j u, j u, L u), \\
\frac{G_{p}(h u, h u, L u)+G_{p}(j u, j u, K u)}{2}
\end{array}\right\} \\
& =\left\{\begin{array}{l}
G_{p}\left(w_{1}, w_{1}, w_{2}\right), G_{p}\left(w_{1}, w_{1}, w_{1}\right), G_{p}\left(w_{2}, w_{2}, w_{2}\right), \\
\frac{G_{p}\left(w_{1}, w_{1}, w_{2}\right)+G_{p}\left(w_{2}, w_{2}, w_{1}\right)}{2}
\end{array}\right\} \\
& =\left\{G_{p}\left(w_{1}, w_{1}, w_{2}\right), \frac{G_{p}\left(w_{1}, w_{1}, w_{2}\right)+G_{p}\left(w_{2}, w_{2}, w_{1}\right)}{2}\right\}
\end{aligned}
$$

If $u_{u, u, u}(h, j, K, L)=\frac{G_{p}\left(w_{1}, w_{1}, w_{2}\right)+G_{p}\left(w_{2}, w_{2}, w_{1}\right)}{2}$, then we get:

$$
\begin{aligned}
G_{p}\left(w_{1}, w_{1}, w_{2}\right) & \leq \frac{q}{2}\left\{G_{p}\left(w_{1}, w_{1}, w_{2}\right)+G_{p}\left(w_{2}, w_{2}, w_{1}\right)\right\} \\
& \leq \frac{q}{2-q} G_{p}\left(w_{2}, w_{2}, w_{1}\right) \\
& =\frac{q}{2-q} G_{p}\left(w_{1}, w_{2}, w_{2}\right)
\end{aligned}
$$

In like manner, we rely on (2) and follow same step to achieve next result:

$G_{p}\left(w_{1}, w_{2}, w_{2}\right) \leq \frac{q}{2-q} G_{p}\left(w_{1}, w_{1}, w_{2}\right)$

Combining (5) and (6) gives:

$$
G_{p}\left(w_{1}, w_{1}, w_{2}\right) \leq\left(\frac{q}{2-q}\right)^{2} G_{p}\left(w_{1}, w_{1}, w_{2}\right)
$$

A contradiction. Thus $w_{1}=w_{2}$ and hence $h u=j u=$ $K u=L u$.

Next, we show that $u$ is the common fixed point of $h$, $j, K$ and $L$. We claim that $u=j u$ :

$$
G_{p}(u, u, j u)=G_{p}(h v, h v, j u) \leq q u_{v, v, u}(h, j, K, L)
$$

where:

$$
\begin{aligned}
& u_{v, v, u}(h, j, K, L) \\
& \in\left\{\begin{array}{l}
G_{p}(K v, K v, L u), G_{p}(h v, h v, K v), G_{p}(j u, j u, L u), \\
\frac{G_{p}(h v, h v, L u)+G_{p}(j u, j u, K v)}{2}
\end{array}\right\} \\
& =\left\{\begin{array}{l}
G_{p}(u, u, u), G_{p}(u, u, u), G_{p}(j u, j u, u), \\
\frac{G_{p}(u, u, u)+G_{p}(j u, j u, u)}{2}
\end{array}\right\} \\
& =\left\{G_{p}(j u, j u, u), \frac{1}{2} G_{p}(j u, j u, u)\right\}
\end{aligned}
$$$$
\text { If } \quad u_{v, v, u}(h, j, K, L)=G_{p}(j u, j u, u) \quad \text { then }
$$$$
G_{p}(u, u, j u) \leq q G_{p}(j u, j u, u)=q G_{p}(u, j u, j u) .
$$$$
\text { If } \quad u_{v, v, u}(h, j, K, L)=\frac{1}{2} G_{p}(j u, j u, u) \quad \text { then }
$$$$
G_{p}(u, u, j u) \leq \frac{q}{2} G_{p}(j u, j u, u)=\frac{q}{2} G_{p}(u, j u, j u) .
$$

Let $\max \left\{q, \frac{q}{2}\right\}=q$ then:

$G_{p}(u, u, j u) \leq q G_{p}(u, j u, j u)$

With the aid of (2) and following the same procedure as above yields the understated: 


$$
G_{p}(u, j u, j u) \leq q G_{p}(j u, u, u)=q G_{p}(u, u, j u)
$$

Combining (7) and (8) gives, $G p(u, j u, j u) \leq q^{2} G_{p}(u, u$, $t$ ), a contradiction.

Hence:

$$
u=h u=j u=K u=L u
$$

Suppose there is a different common fixed point of $h$, $j, K$ and $L$ say $t$ such that $u \neq t$ then using (1) we have:

$$
G_{p}(u, u, t)=G_{p}(h u, h u, j t) \leq q u_{u, u, t}(h, j, K, L)
$$

where:

$$
\begin{aligned}
u_{u, u, t}(h, j, K, L) & \in\left\{\begin{array}{l}
G_{p}(K u, K u, L t), G_{p}(h u, h u, K u), \\
G_{p}(j t, j t, L t), \\
\frac{G_{p}(h u, h u, L t)+G_{p}(j t, j t, K u)}{2}
\end{array}\right\} \\
& =\left\{\begin{array}{l}
G_{p}(u, u, t), G_{p}(u, u, u), G_{p}(t, t, t), \\
\frac{G_{p}(u, u, t)+G_{p}(t, t, u)}{2}
\end{array}\right\} \\
& =\left\{G_{p}(u, u, t), \frac{G_{p}(u, u, t)+G_{p}(t, t, u)}{2}\right\}
\end{aligned}
$$

$u_{u, u, t}(h, j, K, L)=G p(u, u, t)$ then $G p(u, u, t) \leq q G_{p}(u, u, t)$, a contradiction $u_{u, u, t}(h, j, K, L)=\frac{G_{p}(u, u, t)+G_{p}(t, t, u)}{2}$ then:

$$
\begin{aligned}
G_{p}(u, u, t) & \leq \frac{q}{2}\left(G_{p}(u, u, t)+G_{p}(t, t, u)\right) \\
& \leq \frac{q}{2-q} G_{p}(t, t, u)
\end{aligned}
$$

Let $\max \left\{q, \frac{q}{2-q}\right\}=q$ then:

$G_{p}(u, u, t) \leq q G_{p}(t, t, u)=q G_{p}(u, t, t)$

Similarly, we have:

$G_{p}(u, t, t) \leq q G_{p}(u, u, t)$

Combining (9) and (10) yields $G p(u, u, t) \leq q^{2} G_{p}(u, u, t)$, a contradiction since $q<1$. Thus, the uniqueness is proved.

\section{Corollary 2.2}

Let $h, j$ and $L$ be self-maps of a G-partial metric space $A$ satisfying $h(A) \cup j(A) \subset L(A)$ and $G_{p}(h a, h a$, $j b) \leq q u_{a, b, b}(h, j, L)$, where $q \in(0,1)$ and:

$$
u_{a, a, b}(h, j, L) \in\left\{\begin{array}{l}
G_{p}(h a, h a, L b), G_{p}(h a, h a, L a), \\
G_{p}(j b, j b, L b), \\
\frac{G_{p}(h a, h a, L b)+G_{p}(j b, j b, L a)}{2}
\end{array}\right\}
$$

and $G_{p}(h a, h a, j b) \leq q u_{a, b, b}(h, j, L)$ where $q \in(0,1)$ and:

$$
u_{a, b, b}(h, j, K, L) \in\left\{\begin{array}{l}
G_{p}(h a, L b, L b), G_{p}(h a, L a, L a), \\
\frac{G_{p}(j b, L b, L b),}{2}(h a, L b, L b)+G_{p}(j b, L a, L a) \\
2
\end{array}\right\}
$$

for all $a, b \in A$. If one of $h(A), j(A)$ or $L(A)$ is a complete subspace of $A$, then $\{h, L\}$ and $\{j, L\}$ have a unique point of coincidence in $A$. Additionally, if $\{h, L\}$ and $\{j, L\}$ are weakly compatible, then $h, j$ and $L$ have a unique common fixed point.

\section{Theorem 2.3}

Let $h, j, K$ and $L$ be self-maps of a G-partial metric space $A$ satisfying $h(A) \subset L(A), j(A) \subset K(A)$ and:

$$
\begin{aligned}
G_{p}(h a, h a, j b) & \leq p G_{p}(K a, K a, L b)+q G_{p}(h a, h a, K a) \\
& +r G_{p}(j b, j b, L b) \\
& +t\left[G_{p}(h a, h a, L b)+G_{p}(j b, j b, K a)\right]
\end{aligned}
$$

and:

$$
\begin{aligned}
G_{p}(h a, j b, j b) & \leq p G_{p}(K a, L b, L b)+q G_{p}(h a, K a, K a) \\
& +r G_{p}(j b, L b, L b) \\
& +t\left[G_{p}(h a, L b, L b)+G_{p}(j b, K a, K a)\right]
\end{aligned}
$$

for all $a, b \in A$, where $p, q, r, t, \in[0,1)$ satisfy $p+q+r+t<1$.

If one of $h(A), j(A), K(A)$ or $L(A)$ is a complete subspace of $A$, then $\{h, K\}$ and $\{j, L\}$ have a unique point of coincidence in $A$. Additionally, if $\{h, K\}$ and $\{j$, $L\}$ are weakly compatible, then $h, j, K$ and $L$ have a unique common fixed point.

\section{Proof}

For any arbitrary $a_{0} \in A$, we can generate two sequence $\left\{a_{n}\right\}$ and $\left\{b_{n}\right\}$ in $A$ as in the proof of Theorem 1. Then:

$$
\begin{aligned}
& G_{p}\left(b_{n+1}, b_{n+1}, b_{n}\right) \\
& =G_{p}\left(b_{2 k+1}, b_{2 k+1}, b_{2 k}\right) \\
& =G_{p}\left(h a_{2 k}, h a_{2 k}, j a_{2 k-1}\right) \\
& \leq p G_{p}\left(K a_{2 k}, K a_{2 k}, L a_{2 k-1}\right)+q G_{p}\left(h a_{2 k}, h a_{2 k}, K a_{2 k}\right)
\end{aligned}
$$




$$
\begin{aligned}
& +r G_{p}\left(j a_{2 k-1}, j a_{2 k-1}, L a_{2 k-1}\right) \\
& +t\left[G_{p}\left(h a_{2 k}, h a_{2 k}, L a_{2 k-1}\right)+G_{p}\left(j a_{2 k-1}, j a_{2 k-1}, K a_{2 k}\right)\right] \\
& \leq p G_{p}\left(b_{2 k}, b_{2 k}, b_{2 k-1}\right)+q G_{p}\left(b_{2 k+1}, b_{2 k+1}, b_{2 k}\right) \\
& +r G_{p}\left(b_{2 k}, b_{2 k}, b_{2 k-1}\right) \\
& +t\left[G_{p}\left(b_{2 k+1}, b_{2 k+1}, b_{2 k-1}\right)+G_{p}\left(b_{2 k}, b_{2 k}, b_{2 k}\right)\right] \\
& \leq p G_{p}\left(b_{2 k}, b_{2 k}, b_{2 k-1}\right)+q G_{p}\left(b_{2 k+1}, b_{2 k+1}, b_{2 k}\right) \\
& +r G_{p}\left(b_{2 k}, b_{2 k}, b_{2 k-1}\right) \\
& +t\left[G_{p}\left(b_{2 k+1}, b_{2 k+1}, b_{2 k}\right)+G_{p}\left(b_{2 k}, b_{2 k}, b_{2 k-1}\right)\right] \\
& \left.=p G_{p}\left(b_{2 k}, b_{2 k}, b_{2 k}\right)+G_{p}\left(b_{2 k}, b_{2 k}, b_{2 k-1}\right)+q b_{p k}\left(b_{2 k+1}, b_{2 k+1}, b_{2 k}\right)\right] \\
& +r G_{p}\left(b_{2 k}, b_{2 k}, b_{2 k-1}\right) \\
& +t\left[G_{p}\left(b_{2 k+1}, b_{2 k+1}, b_{2 k}\right)+G_{p}\left(b_{2 k}, b_{2 k}, b_{2 k-1}\right)\right] \\
& =p G_{p}\left(b_{n}, b_{n}, b_{n-1}\right)+q G_{p}\left(b_{n+1}, b_{n+1}, b_{n}\right) \\
& +r G_{p}\left(b_{n}, b_{n}, b_{n-1}\right) \\
& +t\left[G_{p}\left(b_{n+1}, b_{n+1}, b_{n}\right)+G_{p}\left(b_{n}, b_{n}, b_{n-1}\right)\right] \\
& \leq(p+r+t) G_{p}\left(b_{n}, b_{n}, b_{n-1}\right) \\
& +(q+t)\left(b_{n+1}, b_{n+1}, b_{n}\right) \\
& \leq \frac{p+r+t}{1-q-t} G_{p}\left(b_{n}, b_{n}, b_{n-1}\right) \\
& \left.+q_{1}\right)
\end{aligned}
$$

If $n$ is odd, so $n=2 k+1$ for some $k \in N$. Then using (11) we obtain:

$$
\begin{aligned}
& G_{p}\left(b_{n+1}, b_{n+1}, b_{n}\right) \\
& =G_{p}\left(b_{2 k+2}, b_{2 k+2}, b_{2 k+1}\right) \\
& =G_{p}\left(h a_{2 k+1}, h a_{2 k+1}, j a_{2 k}\right) \\
& \leq p G_{p}\left(K a_{2 k+1}, K a_{2 k+1}, L a_{2 k}\right) \\
& +q G_{p}\left(h a_{2 k+1}, h a_{2 k+1}, K a_{2 k+1}\right) \\
& +r G_{p}\left(j a_{2 k}, j a_{2 k}, L a_{2 k}\right) \\
& +t G_{p}\left(h a_{2 k+1}, h a_{2 k+1}, L a_{2 k}\right)+G_{p}\left(j a_{2 k}, j a_{2 k}, K a_{2 k+1}\right) \\
& =p G_{p}\left(b_{2 k+1}, b_{2 k+1}, b_{2 k}\right)+q G_{p}\left(b_{2 k+2}, b_{2 k+2}, b_{2 k+1}\right) \\
& +r G_{p}\left(b_{2 k+1}, b_{2 k+1}, b_{2 k}\right) \\
& +t\left[G_{p}\left(b_{2 k+2}, b_{2 k+2}, b_{2 k}\right)+G_{p}\left(b_{2 k+1}, b_{2 k+1}, b_{2 k+1}\right)\right] \\
& \leq p G_{p}\left(b_{2 k+1}, b_{2 k+1}, b_{2 k}\right)+q G_{p}\left(b_{2 k+2}, b_{2 k+2}, b_{2 k+1}\right) \\
& +r G_{p}\left(b_{2 k+1}, b_{2 k+1}, b_{2 k}\right) \\
& +t\left[G_{p}\left(b_{2 k+1}, b_{2 k+1}, b_{2 k+1}\right)+G_{p}\left(b_{2 k+1}, b_{2 k+1}, b_{2 k}\right)\right. \\
& \left.=G_{p}\left(b_{2 k+1}, b_{2 k+1}, b_{2 k+1}\right)+G_{p}\left(b_{2 k+1}, b_{2 k+1}, b_{2 k+1}\right)\right] \\
& +p G_{p}\left(b_{2 k+1}, b_{2 k+1}, b_{2 k}\right)+q G_{p}\left(b_{2 k+2}, b_{2 k+2}, b_{2 k+1}\right) \\
& +r G_{p}\left(b_{2 k+1}, b_{2 k+1}, b_{2 k}\right) \\
& +t\left[G_{p}\left(b_{2 k+2}, b_{2 k+2}, b_{2 k+1}\right)+G_{p}\left(b_{2 k+1}, b_{2 k+1}, b_{2 k}\right)\right] \\
& =p G_{p}\left(b_{n}, b_{n}, b_{n-1}\right)+q G_{p}\left(b_{n+1}, b_{n+1}, b_{n}\right) \\
& +r G_{p}\left(b_{n}, b_{n}, b_{n-1}\right) \\
&
\end{aligned}
$$

where, $\lambda=\frac{p+r+t}{1-q-t}<1$.

Consequently, we obtain $G p\left(b_{n+1}, \quad b_{n+1}\right.$, $\left.b_{n}\right) \leq \lambda^{n} G_{p}\left(b_{1}, b_{1}, b_{0}\right)$.

For $m>n$ and using the rectangle inequality we get:

$$
\begin{aligned}
G_{p}\left(b_{n}, b_{n}, b_{m}\right) \leq & G_{p}\left(b_{n}, b_{n}, b_{n+1}\right)+G_{p}\left(b_{n+1}, b_{n+1}, b_{n+2}\right) \\
& +G_{p}\left(b_{n+2}, b_{n+2}, b_{n+3}\right)+\cdots+G_{p}\left(b_{m-1}, b_{m}, b_{m}\right) \\
& -G_{p}\left(b_{n+1}, b_{n+1}, b_{n+1}\right)-\cdots-G_{p}\left(b_{m-1}, b_{m-1}, b_{m-1}\right) \\
& \leq G_{p}\left(b_{n}, b_{n}, b_{n+1}\right)+G_{p}\left(b_{n+1}, b_{n+1}, b_{n+2}\right) \\
& +G_{p}\left(b_{n+2}, b_{n+2}, b_{n+3}\right)+\cdots+G_{p}\left(b_{m-1}, b_{m}, b_{m}\right) \\
& \leq \lambda^{n} G_{p}\left(b_{0}, b_{0}, b_{1}\right)+\lambda^{n+1} G_{p}\left(b_{0}, b_{0}, b_{1}\right) \\
& +\cdots+\lambda^{m-1} G_{p}\left(b_{0}, b_{0}, b_{1}\right) \\
& \leq\left(\lambda^{n}+\lambda^{n+1}+\cdots+\lambda^{m-1}\right) G_{p}\left(b_{0}, b_{0}, b_{1}\right) \\
& \leq \lambda^{n}\left(1+\lambda+\cdots+\lambda^{m-n-1}\right) G_{p}\left(b_{0}, b_{0}, b_{1}\right) \\
& \leq \frac{\lambda^{n}}{1-\lambda} G_{p}\left(b_{0}, b_{0}, b_{1}\right)
\end{aligned}
$$

This implies that $G_{p}\left(b_{n}, b_{n}, b_{m}\right) \rightarrow 0$ as $m, n \rightarrow \infty$. Thus $\left\{b_{n}\right\}$ is a Cauchy sequence in $A$. Suppose $K(A)$ such that $K a_{2 k}=b_{2 k} \rightarrow u$ as $k \rightarrow \infty$. It is equivalent to $K a_{2 k}=j a_{2 k-1}=$ $b_{2 k} \rightarrow u$ and $b_{2 k-1}=L a_{2 k-1}=h a_{2 k-2} \rightarrow u$ as $k \rightarrow \infty$. Also $G_{p}(u, u, u)=\lim _{k \rightarrow \infty} G_{p}\left(b_{2 k}, u, u\right)=\lim _{m, n \rightarrow \infty} G_{p}\left(b_{n}, b_{n}, b_{m}\right)=0$.

Consequently, we can find a $v$ in $A$ such that $K v=u$. We claim that $h v=u$. On the contrary we have:

$$
\begin{aligned}
G_{p}(h v, h v, u) & \leq G_{p}\left(h v, h v, j a_{2 k-1}\right)+G_{p}\left(j a_{2 k-1}, j a_{2 k-1}, u\right) \\
& -G_{p}\left(j a_{2 k-1}, j a_{2 k-1}, j a_{2 k-1}\right) \\
& \leq G_{p}\left(h v, h v, j a_{2 k-1}\right)+G_{p}\left(j a_{2 k-1}, j a_{2 k-1}, u\right) \\
& \leq p G_{p}\left(K v, K v, L a_{2 k-1}\right)+q G_{p}(h v, h v, K v) \\
& +r G_{p}\left(j a_{2 k-1}, j a_{2 k-1}, L a_{2 k-1}\right) \\
& +t\left[G_{p}\left(h v, h v, L a_{2 k-1}\right)+G_{p}\left(j a_{2 k-1}, j a_{2 k-1}, K v\right)\right] \\
& +G_{p}\left(j a_{2 k-1}, j a_{2 k-1}, u\right)
\end{aligned}
$$

As $k \rightarrow \infty$ we obtain: 


$$
\begin{aligned}
G_{p}(h v, h v, u) & \leq p G_{p}(K v, K v, u)+q G_{p}(h v, h v, K v) \\
& +r G_{p}(u, u, u) \\
& +t\left[G_{p}(h v, h v, u)+G_{p}(u, u, K v)\right] \\
& +G_{p}(u, u, u) \\
& \leq q G_{p}(h v, h v, u)+t G_{p}(h v, h v, u) \\
& \leq(q+t) G_{p}(h v, h v, u)
\end{aligned}
$$

A contradiction, hence $h v=u$.

Since $h(A) \subset L(A)$, it implies that $u \in L(A)$. Therefore, we can find a $w$ in $A$ such that $L w=u$. Thus using (1) we shall show that $j w=u$ :

$$
\begin{aligned}
G_{p}(h v, h v, u) & \leq G_{p}\left(j w, j w, h a_{2 k}\right)+G_{p}\left(h a_{2 k}, h a_{2 k}, u\right) \\
& -G_{p}\left(h a_{2 k}, h a_{2 k}, h a_{2 k}\right) \\
& \leq G_{p}(j w, j w, v)+G_{p}\left(h a_{2 k}, h a_{2 k}, u\right) \\
& =G_{p}\left(h a_{2 k}, j w, j w\right)+G_{p}\left(u, h a_{2 k}, h a_{2 k}\right) \\
& \leq p G_{p}\left(h a_{2 k}, h a_{2 k}, L w\right)+q G_{p}\left(h a_{2 k}, h a_{2 k}, K a_{2 k}\right) \\
& +r G_{p}(j w, j w, L w) \\
& +t\left[G_{p}\left(h a_{2 k}, h a_{2 k}, L w\right)+G_{p}\left(j w, j w, K a_{2 k}\right)\right]
\end{aligned}
$$

As $k \rightarrow \infty$ we obtain:

$$
\begin{aligned}
G_{p}(j w, j w, u) & \leq p G_{p}(u, u, L w)+q G_{p}(u, u, u) \\
& +r G_{p}(j w, j w, u) \\
& +t\left[G_{p}(u, u, L w)+G_{p}(j w, j w, u)\right] \\
& =r G_{p}(j w, j w, u)+t G_{p}(j w, j w, u) \\
& =(r+t) G_{p}(j w, j w, u)
\end{aligned}
$$

A contradiction, hence $j w=u$. Therefore $K v=h v=$ $L w=j w=u$.

Since $\{h, K\}$ and $\{j, L\}$ are weakly compatible then we have, $K u=\mathrm{K} h v=j K v=h u=w_{1}$ and $j u=j L w$ $=L j w=L u=w_{2}$.

We shall show that $w_{1}=w_{2}$ :

$$
\begin{aligned}
G_{p}\left(w_{1}, w_{2}, w_{3}\right) & =G_{p}(h u, h u, j u) \\
& \leq G_{p}(K u, K u, L u)+q G_{p}(h u, h u, K u) \\
& +r G_{p}(j u, j u, L u) \\
& +t\left[G_{p}(h u, h u, L u)+G_{p}(j u, j u, K u)\right] \\
& =p G_{p}\left(w_{1}, w_{1}, w_{2}\right)+q G_{p}\left(w_{1}, w_{1}, w_{1}\right) \\
& +r G_{p}\left(w_{2}, w_{2}, w_{2}\right) \\
& +t\left[G_{p}\left(w_{1}, w_{1}, w_{2}\right)+G_{p}\left(w_{2}, w_{2}, w_{1}\right)\right] \\
& =p G_{p}\left(w_{1}, w_{1}, w_{2}\right) \\
& +t\left[G_{p}\left(w_{1}, w_{1}, w_{2}\right)+G_{p}\left(w_{2}, w_{2}, w_{1}\right)\right] \\
& =(p+t) G_{p}\left(w_{1}, w_{1}, w_{2}\right)+t G_{p}\left(w_{2}, w_{2}, w_{1}\right) \\
& \leq \frac{t}{1-p-t} G_{p}\left(w_{2}, w_{2}, w_{1}\right) \\
& \leq z G_{p}\left(w_{1}, w_{1}, w_{2}\right)
\end{aligned}
$$

where:

$$
z=\frac{t}{1-p-t}<1
$$

Using (12) we have:

$$
\begin{aligned}
G_{p}\left(w_{1}, w_{2}, w_{2}\right) & =G_{p}(h u, j u, j u) \\
& \leq p G_{p}(K u, L u, L u)+q G_{p}(h u, K u, K u) \\
& +r G_{p}(j u, L u, L u) \\
& +t\left[G_{p}(h u, L u, L u)+G_{p}(j u, K u, K u)\right] \\
& =p G_{p}\left(w_{1}, w_{2}, w_{2}\right)+q G_{p}\left(w_{1}, w_{1}, w_{1}\right) \\
& +r G_{p}\left(w_{2}, w_{2}, w_{2}\right) \\
& +t\left[G_{p}\left(w_{1}, w_{2}, w_{2}\right)+G_{p}\left(w_{2}, w_{1}, w_{1}\right)\right] \\
& =p G_{p}\left(w_{1}, w_{2}, w_{2}\right) \\
& +t\left[G_{p}\left(w_{1}, w_{2}, w_{2}\right)+G_{p}\left(w_{2}, w_{1}, w_{1}\right)\right] \\
& =(p+t) G_{p}\left(w_{1}, w_{2}, w_{2}\right)+t G_{p}\left(w_{2}, w_{1}, w_{1}\right) \\
& \leq \frac{t}{1-p-t} G_{p}\left(w_{2}, w_{1}, w_{1}\right) \\
& \leq z G_{p}\left(w_{1}, w_{1}, w_{2}\right)
\end{aligned}
$$

where:

$$
z=\frac{t}{1-p-t}<1
$$

Combining (15) and (16) yields:

$$
G_{p}\left(w_{1}, w_{1}, w_{2}\right) \leq z G_{p}\left(w_{1}, w_{2}, w_{2}\right) \leq z^{2} G_{p}\left(w_{1}, w_{1}, w_{2}\right)
$$

This implies that $G p\left(w_{1}, w_{1}, w_{2}\right)=0$. Hence $w_{1}=w_{2}$.

Since the point of coincidence is unique we have $K u=h u=L u=j u$. Now we shall show that $u=j u$. Using (11):

$$
\begin{aligned}
G_{p}(u, u, j u) & =G_{p}(h u, h u, j u) \\
& \leq p G_{p}(K u, K u, L u)+q G_{p}(h u, h u, K u) \\
& +r G_{p}(j u, j u, L u) \\
& +t\left[G_{p}(h u, h u, L u)+G_{p}(j u, j u, K u)\right] \\
& =p G_{p}(u, u, u)+q G_{p}(u, u, u) \\
& +r G_{p}(j u, j u, L u) \\
& +t\left[G_{p}(u, u, u)+G_{p}(j u, j u, K u)\right] \\
& =(r+t) G_{p}(j u, j u, u)
\end{aligned}
$$




$$
\begin{aligned}
G_{p}(u, u, j u) & =G_{p}(h u, j u, j u) \\
& \leq p G_{p}(K u, L u, L u)+q G_{p}(h u, K u, K u) \\
& +r G_{p}(j u, L u, L u) \\
& +t\left[G_{p}(h u, L u, L u)+G_{p}(j u, K u, K u)\right] \\
& =p G_{p}(u, u, u)+q G_{p}(u, u, u) \\
& +r G_{p}(j u, u, u) \\
& +t\left[G_{p}(u, u, u)+G_{p}(j u, u, u)\right] \\
& =(r+t) G_{p}(j u, u, u)=(r+t) G_{p}(u, u, j u)
\end{aligned}
$$

From (17) and (18) we obtain $G p(u, u$, $j u) \leq(r+t)^{2} G p(u, u, j u)$.

Since $(r+t)<1$ then $G p(u, u, j u)=0$. Thus $j u=u ; u$ is the common fixed point of $h, j, K$ and $L$. The uniqueness of the common fixed point of $h, j, K$ and $L$ is proveable.

\section{Corollary 2.4}

Let $h$ and $L$ be self-maps of a G-partial metric space $A$ satisfying $h(A) \subset L(A)$ and:

$$
\begin{aligned}
G_{p}(h a, h a, h b) & \leq p G_{p}(L a, L a, L b)+q G_{p}(h a, h a, L a) \\
& +r G_{p}(h b, h b, L b) \\
& +t\left[G_{p}(h a, h a, L b)+G_{p}(h b, h b, L a)\right]
\end{aligned}
$$

and

$$
\begin{aligned}
G_{p}(h a, h b, h b) & \leq p G_{p}(L a, L b, L b)+q G_{p}(h a, h a, L a) \\
& +r G_{p}(h b, L b, L b) \\
& +t\left[G_{p}(h a, L b, L b)+G_{p}(h b, L a, L a)\right]
\end{aligned}
$$

for all $a, b \in A$, where $p, q, r, t \in[0,1)$ satisfy $p+q+r+t<1$.

If one of $h(A)$ or $L(A)$ is a complete subspace of $A$, then $h$ and $L$ have a unique point of coincidence in $A$. Moreover if $h$ and $L$ are weakly compatible, then $h$ and $L$ have a unique common fixed point.

\section{Conclusion}

We prove that the common fixed point exist and it's unique for four maps which satisfy some generalized contractive maps in G-partial metric spaces. We avoided the use of any map to arrive at our outcome.

\section{Acknowledgement}

The authors appreciate the management of Covenant University for the financial support.

\section{Author's Contributions}

Kanayo Stella Eke: Conceptualized the idea, prepared and developed the article.

Grace O. Akinlabi: Proofread and typed it.

\section{Ethics}

We are wholly responsible for this article; no ethical query is expected thereof.

\section{References}

Abbas, M. and B.E. Rhoades, 2009. Common fixed point results for noncommuting mappings without continuity in generalized metric spaces. Applied Math. Comput., 215: 262-269. DOI: 10.1016/j.amc.2009.04.085

Abbas, M., B.E. Rhoades and T. Nazir, 2010. Common fixed points for four maps in cone metric spaces. Applied Math. Comput., 216: 80-86. DOI: 10.1016/j.amc.2010.01.003

Eke, K.S. and J.O. Olaleru, 2013. Some fixed point results on ordered G-partial metric spaces. ICASTOR J. Math. Sci., 7: 65-78.

Eke, K.S., 2015. Some fixed and coincidence point results for expansive mappings on G-partial metric spaces. Adv. Fixed Point Theory, 5: 369-386.

Jungck, G., 1976. Commuting mappings and fixed points. Am. Math. Monthly, 83: 261-263. DOI: $10.2307 / 2318216$

Jungck, G., 1986. Compatible mappings and common fixed points. Int. J. Math. Sci., 9: 771-779. DOI: $10.1155 / \mathrm{S} 0161171286000935$

Jungck, G., 1996. Common fixed points for noncontinuous nonself maps on non-metric spaces. Far East J. Math. Sci., 4: 199-215.

Matthews, S.G., 1992. Partial Metric Spaces. Department of Computer Science, University of Warwick.

Mustafa, Z. and B. Sims, 2006. A new approach to generalized metric spaces. J. Nonlinear Convex Analysis, 7: 289-297.

Olaleru, J.O., K.S. Eke and H. Olaoluwa, 2014. Some fixed point results for Ciric-type contractive mappings in ordered G-partial metric space. Applied Math., 5: 1004-1012. DOI: 10.4236/am.2014.56095

Sessa, S., 1982. On a weak commutativity condition of mappings in fixed point considerations. Publicat. Inst. Math. Soc., 32: 95-105. 